\title{
Electrodiffusion of Lipids on Membrane Surfaces
}

\author{
Y. C. Zhou* \\ Department of Mathematics, Colorado State University, Fort Collins, CO 80523, USA
}

(Dated: October 13, 2018)

\begin{abstract}
Lateral translocation of lipids and proteins is a universal process on membrane surfaces. Local aggregation or organization of lipids and proteins can be induced when the random lateral motion is mediated by the electrostatic interactions and membrane curvature. Although the lateral diffusion rates of lipids on membranes of various compositions are measured and the electrostatic free energies of predetermined protein-membrane-lipid systems can be computed, the process of the aggregation and the evolution to the electrostatically favorable states remain largely undetermined. Here we propose an electrodiffusion model, based on the variational principle of the free energy functional, for the self-consistent lateral drift-diffusion of multiple species of charged lipids on membrane surfaces. Finite sizes of lipids are modeled to enforce the geometrical constraint of the lipid concentration on membrane surfaces. A surface finite element method is developed to appropriate the Laplace-Beltrami operators in the partial differential equations (PDEs) of the model. Our model properly describes the saturation of lipids on membrane surfaces, and correctly predicts that the MARCKS peptide can consistently sequester three multivalent phosphatidylinositol 4,5-bisphosphate $\left(\mathrm{PIP}_{2}\right)$ lipids through its basic amino acid residues, regardless of a wide range of the percentage of monovalent phosphatidylserine (PS) in the membrane.
\end{abstract}

\section{INTRODUCTION}

Lateral motion of orientated lipids is an intrinsic membrane property. Through specific spatial and temporal variations of their lateral distribution the lipids regulate many essential membrane-related physiological processes. Multivalent phosphatidylinositol 4,5-bisphosphate $\left(\mathrm{PIP}_{2}\right)$, for instance, plays a central role in anchoring proteins to plasma membranes and in regulating many ion channels, enzymes and cytoskeletons [1-4]. Charged lipids are also involved in driving membrane curvature that is required by cell endocytosis and exocytosis, as illustrated by the lateral movement of monovalent dioleoylphosphatidylserine (DOPS) to favor electrostatic interactions between negatively charged high-curvature membranes and positively charged concave surfaces of Bin/amphiphysin/Rvs (BAR) proteins $[5,6]$. On the other hand, the lateral distribution of lipids are strongly affected by the interactions among lipid molecules, binding proteins, membrane curvature and membrane potential, and the ionic distributions in the solution [5, 7-10]. It is therefore of great importance to study the lateral distribution of lipids under a wide range of physiological conditions to provide qualitative or quantitative information of related physiological processes.

Lateral diffusion is a well recognized model of in-plane lipid motion [11-13]. Various techniques such as fluorescence recovery after photobleaching (FRAP), nuclear magnetic resonance (NMR), electron spin resonance (ESR), and fluorescence spectroscopy have been developed to determine the lateral diffusion coefficients of lipids [14-17], mostly by fitting the measured lengths of random walks to the linear dependence relation $\left\langle r^{2}(t)\right\rangle=D t$ of normal diffusion to determine the the diffusion coefficient $D$. Surprisingly, the measured coefficients can differ by 2 orders of magnitude depending on the particular techniques, materials and environments (in vitro or in vivo, for example) in experiments [18-20]. Furthermore, diffusion in membranes in vivo may not follow the normal linear relation because the membrane contains a number of non-lipid components of different dimensions, which make the diffusion abnormal. According to the classical free-volume diffusion theory of Cohen and Turnbull [21-23], lateral diffusion indeed follows a three-step procedure: (i) creation of a local free volume due to spontaneous thermal fluctuations; (ii) hopping of a diffusive molecule into the void; and (iii) filling of the hole left behind the hopping molecule by another molecule. Along with other attempts to describe the lipid diffusion in real complex membrane systems [24], this theory has been integrated with the scaled-particle theory [25] to model the dependence of the diffusion coefficient on the sizes of lipids and non-lipid components [26], and the theoretical results matched the experiments better than the original free-volume diffusion theory. Recent molecular dynamics (MD) simulations of membrane dynamics have provided different insights of the mechanism of lateral diffusion of lipids. It was shown that the motion of neighboring lipids are strongly correlated, therefore motion of lipids in clusters is more favored over the hopping of individual lipids [27].

Applications of these measured lateral diffusion coefficients to computational simulations or quantitative analysis of physiological processes, however, are scarce, probably due to a lack of appropriate models of the mechanisms that can lead to the variation of the lateral distribution of lipids. Suppose that the lateral diffusion of lipids in a bounded plenary 2-D domain $\Omega$ is described by the standard diffusion equation with source

$$
\frac{\partial \rho}{\partial t}=D \Delta \rho+f(x, y)
$$

where $D$ is the constant diffusion coefficient, then the variation of the continuous lipid concentration $\rho$ can be caused by its initial non-uniform distribution, the product function 
$f(x, y)$ that models the addition or removal of lipids in the domain, or the prescribed distribution of $\rho$ on the boundary of $\Omega$. None of these three features have been well modeled for lipid diffusion. We note, however, that similar models are developed for lateral diffusion of membrane proteins. For instance the aggregation of proteins to the growing clusters in membranes is modeled as an absorbing boundary condition on the moving boundary [28, 29]. For lateral diffusion of lipids or proteins on curved biological membranes one needs to replace the Laplace operator in Eq.(1) by the Laplace-Beltrami operator $\Delta_{s}$ so the effects of surface curvatures on the diffusion rate can be incorporated [30-32]. Such a model also enables us to investigate the long-term effects of the random fluctuations of the surface metric on the lateral diffusion [33]. More importantly, membrane proteins and many regulatory lipids such as PIP $_{2}$ and DOPS are charged hence a drift-diffusion equation is more suitable than the standard diffusion equation (1). Electrodiffusion of charge particles in solution has been extensively studied in past decades, and there has been resurgent interests recently in modifying the classical Poisson-Nernst-Planck (PNP) theory to better model the drift-diffusion of charged particles with finite sizes and particle-particle correlations [34, 35]. PNP theory and its modifications have been extended recently to study the lateral motion of lipids or proteins in membranes. For example, 2-D Poisson-Boltzmann-Nernst-Planck equations with size exclusion are derived from a constraint free energy minimization to study the lateral motion of lipids in the membrane [36], assuming the co-existence of mobile ions, peptides, and lipids in the hydrated leaflets of the membrane. Formation and disassociation of peptide-lipid complexes are modeled via reaction terms in the NernstPlanck equations, and results are compared favorably to their dynamic Monte Carlo simulations [37]. 2-D PNP equations without size exclusion were used to simulate the electrodiffusion of lipids on a planar surface [38], coupled with a nonlinear 3-D Poisson-Boltzmann equation with a charge density neglecting the contribution of charged lipids. While various degrees of agreement to the experimental measurements or other theoretical predictions have been achieved with these models, an electrodiffusion model similar to the modified Poisson-Nernst-Planck equations in bulk are necessary for quantitative study of self-consistent distributions of charged particles on general curved surfaces.

In this paper we developed a generic surface driftdiffusion equation based on a generalized Borukhov model $[35,39]$ to model the electrostatic mediated lateral motion of charged lipids on arbitrarily curved membrane surfaces. The bilayer membrane is described as a dielectric continuum with a constant permittivity and continuous distributions of surface charges, the latter model the distributions of charged lipids on membrane surfaces. The lipids are treated as diffusive hard disks, and their effective radius are taken into account together with the finite sizes of mobile ions in the entropic contribution of the total free energy. Without proper modeling of the lipid sizes the lateral concentration of charged lipids can be easily overestimated wherever there are strong attractive electrostatic interactions, even though the molecular dimensions of lipids have been considered in the determination of the lateral diffusion coefficient. We derive a system of nonlinear partial differential equations (PDEs) and develop the related numerical algorithms. Numerical simulations are carried out to study the surface electrodiffusion of $\mathrm{PIP}_{2}$ due to the electrostatic interactions between the membrane and the MARCKS protein under a wide range of physiological conditions. Our results are well validated by experimental measurements and atomistic modeling of lipid sequestration.

\section{MATHEMATICAL MODELS OF SURFACE ELECTRODIFFUSION}

We consider a solvated protein-membrane system as illustrated in Figure 1(A). In the spirit of the classical fluid density functional theory $[35,36,40]$ we define the following free energy for the membrane-protein-ionic solution system:

$$
\begin{gathered}
F=k_{B} T \int_{\Omega_{s}} \sum_{i=0} \rho_{i}\left[\ln \left(\frac{\rho_{i}}{\xi_{i}}\right)-1\right] d x+k_{B} T \int_{S_{t} \cup S_{b}} \sum_{j=0} \rho_{j}^{l}\left[\ln \left(\frac{\rho_{j}^{l}}{\xi_{j}^{l}}\right)-1\right] d s+ \\
\int_{\Omega}-\frac{1}{2} \epsilon|\nabla \phi|^{2}+\left(\rho^{f}+\lambda \sum_{i=1} z_{i} e \rho_{i}+\sum_{j=1} z_{j}^{l} e \rho_{j}^{l} \delta\left(x-X_{S_{t} \cup S_{b}}\right)\right) \phi d x
\end{gathered}
$$

where $\rho_{i}$ is the ion concentration of the $i^{\text {th }}$ species and $\rho_{j}^{l}$ is the lipid concentration of $j^{\text {th }}$ species, with $i=0$ denotes the neutral solvent (water) molecules and $j=0$ denotes the neutral species of lipids. The characteristic function $\lambda$ is 1 in the solution domain $\Omega_{s}$ and zero elsewhere. $X$ is the coordinates of the membrane surfaces $S_{t}$ and $S_{b} . k_{B}, T, \phi$ are the Boltzmann constant, temperature, and the electrostatic potential, respectively. $z_{i}, z_{j}^{l}$ are the valences of cor- 
responding ions and lipids, and $e$ is the elementary charge. $\rho^{f}$ is the permanent charges in proteins.

$$
\xi_{i}=\frac{1}{a_{i}^{3}}
$$

is the fugacity of the $i^{\text {th }}$ species of ions with effective size $a_{i}$, and

$$
\xi_{j}=\frac{1}{\left(a_{j}^{l}\right)^{2}}
$$

is the fugacity of $j^{\text {th }}$ species of lipids approximated as hard disks with an effective size $a_{j}^{l}[35,41]$. Here the solvent molecules and neutral lipids have respective effective sizes $a_{0}$ and $a_{0}^{l}$, and their concentrations $\rho_{0}, \rho_{0}^{l}$ follow the relations

$$
\begin{array}{r}
\rho_{0} a_{0}^{3}+\sum_{i=1} \rho_{i} a_{i}^{3}=1, \\
\rho_{0}^{l}\left(a_{0}^{l}\right)^{2}+\sum_{j=1} \rho_{j}^{l}\left(a_{j}^{l}\right)^{2}=1 .
\end{array}
$$

These two constraints are enforced directly in the variations of $F$ with respect to $\rho_{i}$ and $\rho_{i}^{l}$ without resorting to a Lagrangian multiplier, in contrast to [36], giving rise to the electrochemical potentials $\mu_{i}$ for ions $(i \geq 1)$ and $\mu_{j}^{l}$ for charged lipids $(j \geq 1)$ :

$$
\begin{aligned}
& \mu_{i}=\frac{\delta F}{\delta \rho_{i}}=z_{i} e \phi+k_{B} T\left[\ln \left(\rho_{i} a_{i}^{3}\right)-k_{i} \ln \left(1-\sum_{k=1} \rho_{k} a_{k}^{3}\right)\right], \\
& \mu_{j}^{l}=\frac{\delta F}{\delta \rho_{j}^{l}}=z_{j}^{l} e \phi+k_{B} T\left[\ln \left(\rho_{j}^{l}\left(a_{j}^{l}\right)^{2}\right)-k_{j}^{l} \ln \left(1-\sum_{k=1} \rho_{k}^{l}\left(a_{k}^{l}\right)^{2}\right)\right],
\end{aligned}
$$

where $k_{i}=\left(a_{i} / a_{0}\right)^{3}, k_{j}^{l}=\left(a_{j}^{l} / a_{0}^{l}\right)^{2}$. These electrochemical potentials are related to the flux of ions and lipids through the constitutive relations for fluxes

$$
J_{i}=-m_{i} \rho_{i} \nabla \mu_{i}, \quad J_{j}^{l}=-m_{j}^{l} \rho_{j}^{l} \nabla_{s} \mu_{j}^{l},
$$

where $\nabla_{s}$ is the surface gradient operator. $m_{i}$ and $m_{j}^{l}$ are the mobilities of respective species of ions and lipids; they are related to the diffusivities $D_{i}$ or $D_{j}^{l}$ through Einstein's relation

$$
D_{i}=m_{i} k_{B} T \quad \text { or } \quad D_{j}^{l}=m_{j}^{l} k_{B} T .
$$

The drift-diffusion equations for ions are obtained from the mass conservation in solution:

$$
\begin{aligned}
& \frac{\partial \rho_{i}}{\partial t}=-\nabla \cdot J_{i}=\nabla \cdot\left(m_{i} \rho_{i} \nabla \mu_{i}\right) \\
& =\nabla \cdot D_{i}\left(\nabla \rho_{i}+\frac{k_{i} \rho_{i} \sum_{p=1} a_{p}^{3} \nabla \rho_{p}}{1-\sum_{p=1} a_{p}^{3} \rho_{p}}+\frac{1}{k_{B} T} \rho_{i} z_{i} e \nabla \phi\right),
\end{aligned}
$$

and the surface drift-diffusion equations for lipids are obtained from the mass conservation on the membrane surfaces if it evolves at a given velocity field $u_{s}$ [42]:

$$
\begin{aligned}
\frac{\partial \rho_{j}^{l}}{\partial t}+\nabla_{s} \cdot\left(\rho_{j}^{l} u_{s}\right)+\rho_{j}^{l}\left(\nabla_{s} \cdot n\right)\left(u_{s} \cdot n\right) & =-\nabla_{s} \cdot J_{j}^{l}=\nabla_{s} \cdot\left(m_{j}^{l} \rho_{j}^{l} \nabla_{s} \mu_{j}^{l}\right) \\
& =\nabla_{s} \cdot D_{j}^{l}\left(\nabla_{s} \rho_{j}^{l}+\frac{k_{j}^{l} \rho_{j}^{l} \sum_{p=1}\left(a_{p}^{l}\right)^{2} \nabla_{s} \rho_{p}^{l}}{1-\sum_{p=1}\left(a_{p}^{l}\right)^{2} \rho_{p}^{l}}+\frac{1}{k_{B} T} \rho_{j}^{l} z_{j}^{l} e \nabla_{s} \phi\right),
\end{aligned}
$$


where $n$ is the outer normal vector of the surface. If there are only two types of lipids of the same effective size, one charged and the other neutral, in a macroscopically static membrane, one can solve only one surface drift-diffusion equation, which is now simplified to be

$\frac{\partial \rho^{l}}{\partial t}=\nabla_{s} \cdot D^{l}\left(\nabla_{s} \rho^{l}+\frac{k^{l}\left(a^{l}\right)^{2} \rho^{l} \nabla_{s} \rho^{l}}{1-\left(a^{l}\right)^{2} \rho_{p}}+\frac{1}{k_{B} T} \rho^{l} z^{l} e \nabla_{s} \phi\right)$,

in which the subscription $j$ for species is no longer needed. For enclosed membrane surfaces Equations (8) and (9) do not have boundary conditions, but both are subject to the constraint of mass conservation

$$
\int_{S} \rho_{j}^{l} d s=T_{j}
$$

where $T_{j}$ is the given total quantity of a species of charged lipids on the surface $S$. Extremization of $F$ with respect to $\phi$ using variation $\delta F / \delta \phi$ gives the Poisson equation for the electrostatic potential $\phi$ :

$$
-\nabla \cdot(\epsilon \nabla \phi)=\rho^{f}+\lambda \sum_{i=1} \rho_{i} z_{i} e \quad \text { in } \Omega,
$$

with interface conditions

$$
\begin{array}{r}
\epsilon_{m b} \frac{\partial \phi_{m b}}{\partial n}=\epsilon_{s} \frac{\partial \phi_{s}}{\partial n}+\sum_{j=1} \rho_{j}^{l} z_{j}^{l} e, \quad \phi_{s}=\phi_{m b} \text { on } S_{t} \cup S_{b}, \\
\epsilon_{m} \frac{\partial \phi_{m}}{\partial n}=\epsilon_{s} \frac{\partial \phi_{s}}{\partial n} \quad \phi_{s}=\phi_{m} \text { on } \Gamma,
\end{array}
$$

where the charge density carried by charged lipids on the membrane surfaces is written as an interface condition. The subscript $m, m b, s$ denote the quantities in proteins, membrane, and the solution, respectively.

To acquire an accurate characterization of the diffusion process we will solve the Poisson equation (11) coupled with the time-dependent drift-diffusion equation (7) and the time-dependent surface drift-diffusion equation (8), using a finite element method [35] and a linear surface finite element method [43]. If only the equilibrium distributions of ions and charged lipids are of interest one can instead solve the Poisson equation coupled with steady-state driftdiffusion equation and stead-state surface electrodiffusion equation. Notice the latter,

$$
\nabla_{s} \cdot D_{j}^{l}\left(\nabla_{s} \rho_{j}^{l}+\frac{k_{j}^{l} \rho_{j}^{l} \sum_{p=1}\left(a_{p}^{l}\right)^{2} \nabla_{s} \rho_{p}^{l}}{1-\sum_{p=1}\left(a_{p}^{l}\right)^{2} \rho_{p}^{l}}+\frac{1}{k_{B} T} \rho_{j}^{l} z_{j}^{l} e \nabla_{s} \phi\right)=0
$$

admits a trivial solution $\rho_{j}^{l}=0$ if the constraint (10) is not enforced. To obtain a physical solution that is consistent with this constraint we introduce the decomposition

$$
\rho_{j}^{l}=\bar{\rho}_{j}^{l}+\hat{\rho}_{j}^{l},
$$

where

$$
\bar{\rho}_{j}^{l}=\frac{T_{j}}{|S|}
$$

is the known average concentration of $\rho_{j}^{l}$ on the surface $S$ with total surface area $|S|$. Replacing $\rho_{j}^{l}$ in equation (12) with this decomposition we get

$$
\nabla_{s} \cdot D_{j}^{l}\left(\nabla_{s} \hat{\rho}_{j}^{l}+\frac{k_{j}^{l}\left(\hat{\rho}_{j}^{l}+\bar{\rho}_{p}^{l}\right) \sum_{p=1}\left(a_{p}^{l}\right)^{2} \nabla_{s} \hat{\rho}_{p}^{l}}{1-\sum_{p=1}\left(a_{p}^{l}\right)^{2}\left(\hat{\rho}_{p}^{l}+\bar{\rho}_{p}^{l}\right)}+\frac{1}{k_{B} T} \hat{\rho}_{j}^{l} z_{j}^{l} e \nabla_{s} \phi\right)=-\nabla_{s} \cdot\left(D^{l} \frac{1}{k_{B} T} \bar{\rho}_{j}^{l} z_{j}^{l} e \nabla_{s} \phi\right)
$$

from which the nontrivial variation $\hat{\rho}_{j}^{l}$ and hence the nontrivial $\rho_{j}^{l}$ can be uniquely solved.

\section{SOLVATED MARCKS-MEMBRANE SYSTEM}

The system, as illustrated by Figure 1(A-B), consists of a MARCKS peptide and a membrane vesicle whose interior and exterior radii are $230 \AA$ and $270 \AA$, respec- 


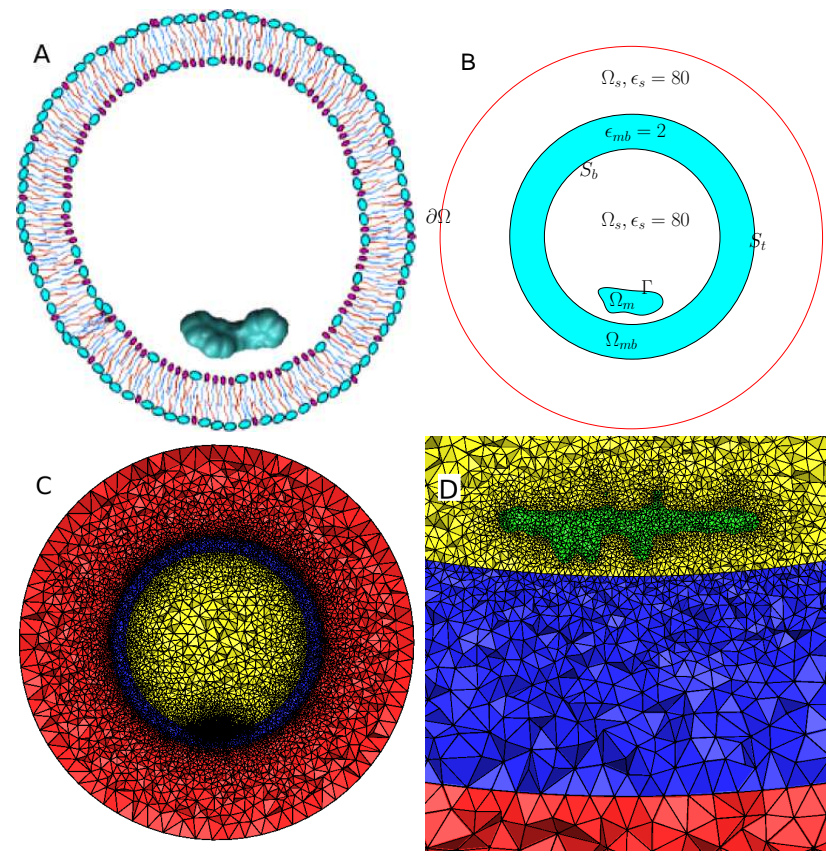

FIG. 1. Solvated MARCKS-membrane system and the computational model. (A) Schematic illustration of the MARCKS peptide residing on the inner leaflet of a vesicle. Lipids with charged (red) and polar (cyan) headgroups are distributed in the membrane. (B) $2 \mathrm{D}$ cross section of the computational model for (A). The domain $\Omega_{m b}$ is the bilayer membrane modeled as a dielectric continuum without atomistic details. Distribution of lipids on membrane surfaces $S_{t}$ and $S_{b}$ follows the surface electrodiffusion equation. Mobile ions are distributed in the aqueous solution exterior and interior to the vesicle. Atomistic structure of the MARCKS peptide is retained and singular charges are distributed in $\Omega_{m}$. $\Gamma$ is the molecuar surface of the peptide, and $\partial \Omega$ is the exterior boundary of the computational domain for the Poisson equation. (C) 3D tetrahedral discretization of the entire computational domain. (D) local refinement of the tetrahedral mesh near the MARCKS peptide.

tively. The MARCKS peptide is unfolded and the peptide is built from the sequence of the effective domain as given in [44]. The peptide is placed above the inner leaflet of the vesicle, the closest distance between the peptide and the membrane surface is about $2 \AA$, with the assumption that the position and orientation are not affected by its interaction with the membrane and lipids. The insertion of MARCKS is not modeled here because the aromatic phenylalanine residues penetrating to the acyl chain region obstruct the diffusion of lipids. Experiments and Monte Carlo simulations suggest that the diffusion coefficients measured on systems free of inserted molecules may not be applicable to the obstructed diffusion $[45,46]$. In order to investigate electrodiffusion of lipids with different valences we will consider two types of lipid compositions, PIP2/PC(Phosphatidylcholine) and PIP2/PS(phosphatidylserine)/PC; all head groups are assumed to have the same effective diameter $8.3 \AA$. The two leaflets of the membrane are assigned the same average lipid composition. The dielectric constant $\epsilon_{m}=\epsilon_{m b}=2$ in the peptide and the membrane, and $\epsilon_{s}=80$ in the solution. There are significant disparities in the measured lateral diffusion coefficients of lipids in the plasma membrane [15, 47], here we choose $D^{l}=580 \AA^{2} / \mu s$. The solution contains the salt $\mathrm{KCl}$ only, whose concentration will be adjusted between $50 \mathrm{mM}$ to $300 \mathrm{mM}$ for the examination of the effects of the salt concentration on the lateral electrodiffusion. The effective sizes of $\mathrm{K}$ and $\mathrm{Cl}$ are $2.7 \AA$ and $3.6 \AA$, respectively, and their diffusion coefficients are $78000 \AA^{2} / \mu$. We note that the final equilibrium distributions of lipids and mobile ions are independent of these diffusion coefficients. There is no ion exchange through the membrane. The Poisson equation (11) is solved in the entire 3-D domain $\overline{\Omega_{s} \cup \Omega_{m b} \cup \Omega_{m}}$, the bulk drift-diffusion equation (7) is solved in the 3-D solvent domain $\Omega_{s}$, and the surface drift-diffusion equation is solved on both spherical membrane surfaces $S_{t}$ and $S_{b}$. This way the lateral diffusion of lipids on two membrane leaflets are coupled. A cross section of the finite element tetrahedral mesh and the locally refined mesh near the peptide are shown in Figure 1(C-D).

\section{COMPUTATIONAL SIMULATIONS AND DISCUSSIONS}

\section{Effects of finite size of $\mathrm{PIP}_{2}$ on its surface distribution}

We compare the aggregation of two models of $\mathrm{PIP}_{2}$ lipids due to the electrostatic attraction of the MARCKS peptide. In one model the lipids are described as particles with vanishing sizes and in the other the lipids have an effective diameter $8.33 \AA$. We observe the aggregation by starting with an initial uniform distribution of $1 \% \mathrm{PIP}_{2}$ on membrane surfaces. The histories of aggregation are shown in Figure 2. The accumulation of charged lipids is found much faster when the size effects are neglected, and its concentration will quickly exceed the upper limit of the lipid concentration, resulting in an unphysical distribution of lipids on membrane surfaces. This upper limit of the concentration of $\mathrm{PIP}_{2}$ (i.e., the membrane consisting of $100 \% \mathrm{PIP}_{2}$ ) is $0.0144 / \AA^{2}$, corresponding to about 36 lipids on a $50 \times 50 \AA^{2}$ membrane surface [5]. The overestimated concentrations of charged lipids can lead to an overestimated dielectric surface force density in studying the protein-membrane electrostatic interactions [48]. In contrast the model with lipid size correction predicts a lipid concentration that is well bounded by the saturation value.

\section{Dependence of PIP $_{2}$ aggregation on its average concentration}

Experiments [1, 2, 4] show that a MARCKS peptide can consistently sequester three or four $\mathrm{PIP}_{2}$ lipids when 


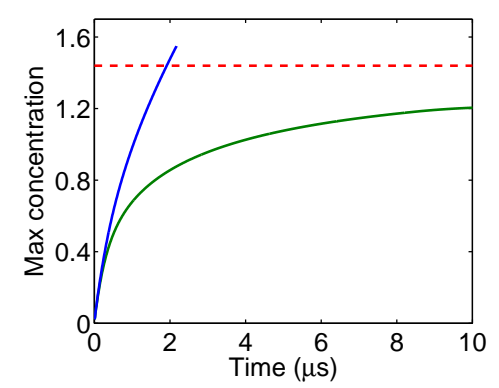

FIG. 2. Without an appropriate model of the lipid size the PIP $_{2}$ quickly exceeds the upper limit of the concentration under the attraction of the MARCKS peptide. Blue: lipids with vanishing size; Green: lipids with an effective diameter $8.33 \AA$. The red line signifies the limit of the lipid concentration $0.0144 / \AA^{2}$. Initial average percentage of $\mathrm{PIP}_{2}$ is $1 \%$. The scale on the vertical axis is amplified 100 -fold.

the average fraction of $\mathrm{PIP}_{2}$ in the membrane varies between $0.01 \%$ and $1 \%$. Suppose $1 \% \mathrm{PIP}_{2}$ is uniformly distributed in the membrane, $3 \mathrm{PIP}_{2}$ lipids shall occupy a surface area of about $144 \times 144 \AA^{2}$. Such an area is nearly 33 times larger than the area coverage of MARCKS peptide $\left(\sim 45 \times 15 \AA^{2}\right)$. For $0.1 \% \mathrm{PIP}_{2}$ this area amounts to almost $1 / 3$ of the total area of the inner leaflet of the vesicle with an inner radius $230 \AA$; and there is barely one $\mathrm{PIP}_{2}$ lipid on the entire inner surface of the vesicle when the membrane contains only $0.01 \% \mathrm{PIP}_{2}$. Consequently we define the lower bound of the $\mathrm{PIP}_{2}$ percentage in our simulations to be $0.1 \%$. The maximum percentage of $\mathrm{PIP}_{2}$ in our simulations is $30 \%$, for which $3 \mathrm{PIP}_{2}$ lipids occupy an area of $26.4 \times 26.4 \AA^{2}$ when distributed uniformly; this area approximates the coverage of MARCKS peptide on the membrane surface. This general assessment of the sparsity of $\mathrm{PIP}_{2}$ lipids suggests that the equilibration of $\mathrm{PIP}_{2}$ distribution in response to the electrostatic perturbation of MARCKS peptide will vary as the initial percentage of $\mathrm{PIP}_{2}$ changes. To quantify these differences and to reveal the continuous dependence of the sequestration of $\mathrm{PIP}_{2}$ on its average concentration we simulate the electrodiffusion of $\mathrm{PIP}_{2}$ with five initial conditions; the results are summarized in Figure 3. With $30 \% \mathrm{PIP}_{2}$ it takes about $0.2 \mu \mathrm{s}$ to re-equilibrate its distribution in response to the approaching of MARCKS peptide, while it takes about $10 \mu \mathrm{s}$ if the membrane contains $1 \% \mathrm{PIP}_{2}$. For an initial percentage of $0.1 \%$ this time becomes as long as $100 \mu \mathrm{s}$. At a low percentage of $\mathrm{PIP}_{2}$ it takes a much longer time for the lipids to move over a larger distance to the vicinity of the MARCKS peptide so the lipids can be sequestered. Cautions must be taken in interpreting this long time of aggregating because the attraction of $\mathrm{PIP}_{2}$ to the peptide is not always started with a uniform distribution of $\mathrm{PIP}_{2}$. Indeed, the MARCKS peptides are recycled in real biological systems: when $\left[\mathrm{Ca}^{2+}\right]$ increase in the local cytoplasm the $\mathrm{Ca}^{2+} /$ calmodulin $(\mathrm{Ca} / \mathrm{CaM})$ will bind to the MARCKS peptides that have attached to the membrane and pull them off. When the detached MARCKS proteins move away from the membrane the sequestered PIP $_{2}$ lipids will be released and diffuse away from the aggregated region [8]. If the period of this recycle is sufficiently small, the reattached MARCKS peptide will quickly attract the $\mathrm{PIP}_{2}$ lipids back before the re-establishment of an uniform distribution of $\mathrm{PIP}_{2}$. It is therefore possible to integrate the current continuum surface electrodiffusion equations for lipids with the diffusion-reaction dynamical models for $\mathrm{Ca}^{2+}$, calmodulins, and other associated molecules to yield an accurate characterization of the biochemical signals that are transmitted through these processes.

We count the number of sequestered $\mathrm{PIP}_{2}$ lipids by integrating the density on the membrane surface

$$
N_{\mathrm{PIP}_{2}}=\int_{S_{p}} \rho^{l} d s
$$

where the sequestration domain $S_{p}$ is defined to be the projection to the inner membrane surface of a $45 \times 15 \AA^{2}$ rectangular centered at Ser162:N, as shown in Figure 3(C). This rectangular is chosen to enclose the entire MARCKS peptide. We note that this definition of sequestration is different from those in the atomistic modeling of lipids [1, 49], where PIP $_{2}$ lipids can be represented as potential surfaces of $-25 \mathrm{mV}$. The sequestration of lipids is identified as the enclosure of these negative potential surfaces by the iso-surfaces of positive potential $(\sim 25 \mathrm{mV})$ that are induced by the basic residues in the peptide. Quantitative agreements are found between the current continuum and the previous atomistic models of lipids. At $0.1 \%$ initial percentage, about 2 lipids will finally be attracted to the membrane surface under the peptide after a long travel, and about $3 \mathrm{PIP}_{2}$ lipids can be sequestered at $1 \%$ initial percentage. More $\mathrm{PIP}_{2}$ lipids are found to be clustered below the peptide with a further increase of its percentage, gradually saturating to the maximum density of lipids. Figure 3(B) indicates that the maximum concentration of $\mathrm{PIP}_{2}$ reaches the saturation density at $30 \%$ of average concentration. Correspondingly there are about $7 \mathrm{PIP}_{2}$ are clustered below the peptide, c.f. Figure 3(D), which account for $72 \%$ of the total lipids in the domain $S_{p}$. It is worth noting that this aggregated distribution of $\mathrm{PIP}_{2}$ is generated from the minimization of the total free energy (2), and is consistent with the electrostatic potential field of the membraneMARCKS system by construction. In contrast, with atomistic models of the sequestration one needs to place charged lipids at positions that are determined a priori so that the electrostatic potential can show favorable electrostatic interactions between basic peptides and phospholipid membranes [50]. The current continuum model thus has a great potential in modeling the membrane-protein systems for which the positions of specific lipids are unknown a priori or change dynamically with the conformational change of membrane or proteins during their interactions. 

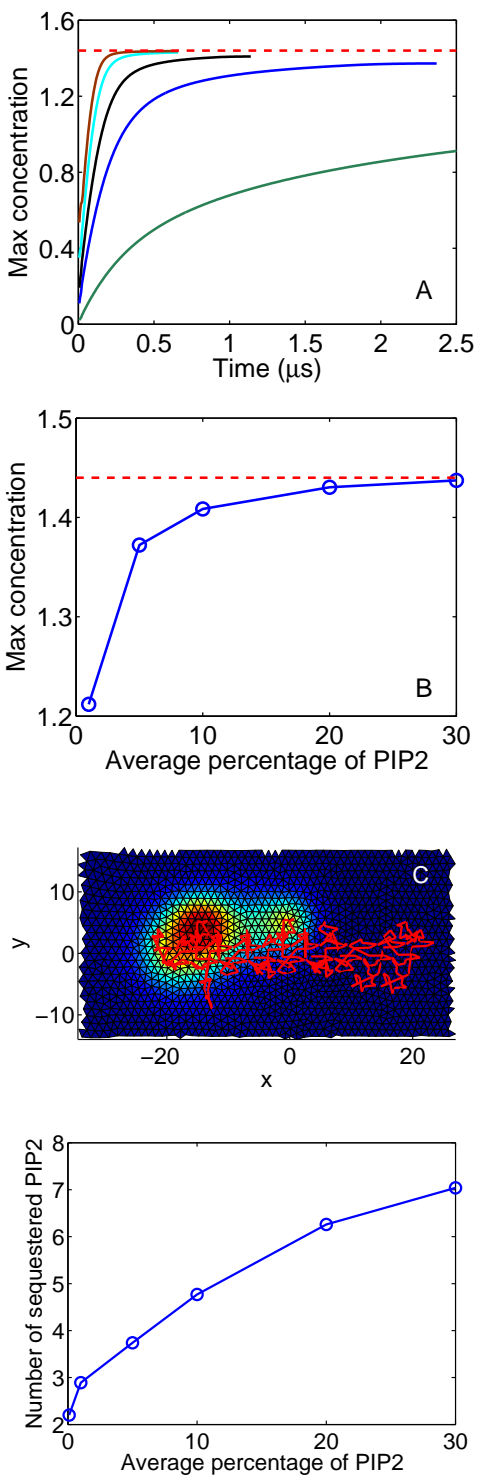

FIG. 3. (A) Accumulation of PIP $_{2}$ near the MARCKS peptide; (B) the dependence of the maximum concentration of $\mathbf{P I P}_{2}$ on the initial average concentration; (C) Integration region used for counting the sequestered $\mathrm{PIP}_{2}$; and (D) Number of sequestered PIP 2 . The initial average concentrations of $\mathrm{PIP}_{2}$ in (A) are $1 \%, 5 \%, 10 \%, 20 \%, 30 \%$ for the green, blue, black, cyan, and maroon lines, respectively. Complete history for the simulation with initial $1 \% \mathrm{PIP}_{2}$ is shown in Figure 2. In (C) the red lines represents the MARCKS peptide above the membrane surface. The integration region is not pre-determined for a conforming triangulation so its boundary is not straight. The average percentages in (D) are $0.1 \%, 1 \%, 5 \%, 10 \%, 20 \%$ and $30 \%$.

\section{Dependence of $\mathrm{PIP}_{2}$ aggregation on the concentration of monovalent lipids}

When there are multiple species of negatively charged lipids on the membrane their concentrations near the MARCKS peptide can be completely different. Boltzmann relation predicts that multivalent lipids such as $\mathrm{PIP}_{2}$ have an affinity that is 1000 times stronger than the monovalent lipids such as phosphatidylserine (PS) when binding to the MARCKS peptide. Although the real distribution of charged lipids on a membrane does not follow the Boltzmann relation due to their large size and correlations, electrostatic free energy of the sequestration showed that $\mathrm{PIP}_{2}$ is favored by the peptide compared to PS [51]. To quantify this competition we simulate electrodiffusion of $\mathrm{PIP}_{2}$ and PS with different fractions. The average percentage of $\mathrm{PIP}_{2}$ is fixed at $1 \%$ and the fraction of PS varies between $1 \%$ and $30 \%$. Figure 4 shows the weak dependence of the aggregation of $\mathrm{PIP}_{2}$ on the fractions of PS that are under investigation. While the total concentrations of $\mathrm{PIP}_{2}$ and PS increase to approach the saturation value with the increase of average concentration of PS, the peak concentration of $\mathrm{PIP}_{2}$ is found decreasing. This decrease is slight nevertheless. The number of sequestered $\mathrm{PIP}_{2}$, computed using equation (1), reaches it smallest value of 2.7 when the average percentage of PS is $30 \%$; the corresponding number of sequestered PS lipids is less than 0.4. These results are consistent with the experimental observations that monovalent acidic lipids are less likely be sequestered by membranebound basic peptides if there are multivalent acidic lipids on the membrane [51], and that the sequestration of $\mathrm{PIP}_{2}$ is more favorable when the mol percentage of monovalent acidic lipids in the membrane decreases [50].

The time dependent solutions of electrodiffusion provides quantitative information that can not be attained through the investigation of electrostatic free energy only. An examination of Figures 4(C-E) shows that there is an increase of monovalent PS lipids for the first few microseconds, in contrast to the continuous growth of the concentration of multivalent $\mathrm{PIP}_{2}$. The strength of positive electrostatic potential near the sequestration region is reduced due to the accumulation of negatively charged lipids. It appears that the monovalent PS lipids are more susceptible to this local change potential so their concentration starts to decrease after a maximum value is reached. This temporary increase of monovalent PS lipids is negligibly small when their average percentage is low, c.f. Figure 4(A).

\section{Dependence of $\mathrm{PIP}_{2}$ aggregation on the ion concentration}

Experiment observations suggest that the aggregation of charged lipids depends on the local electrostatic field [49, 52], whose strength can change drastically with the ion concentration in the solution. This dependence is modeled in the current work through the coupling of the surface electrodiffusion equation with the Poisson equation (11) that admits the ion concentrations determined by the modified Nernst-Planck equations (7). To validate the model we simulate the $\mathrm{PIP}_{2}$ aggregation with $\mathrm{KCl}$ concentration ranging from $50 \mathrm{mM}$ to $300 \mathrm{mM}$. Figure 5 illustrates that $\mathrm{PIP}_{2}$ lipids are less aggregated for large salt concentrations, as a result of the electrostatic screening and the weakened 

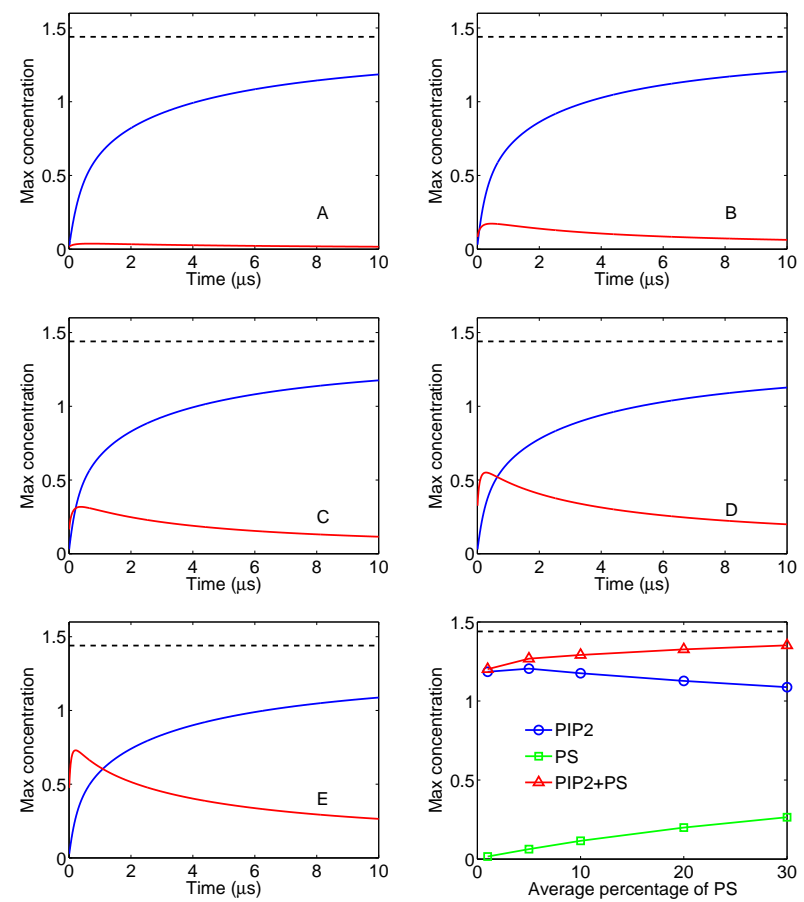

FIG. 4. (A-E) Accumulation of PIP $\mathbf{P}_{2}$ and PS near the MARCKS peptide and $(F)$ the dependence of the maximum concentrations of PIP $_{2}$ and PS on the initial average concentration of PS. The initial average concentrations of PS in (A-E) are $1 \%, 5 \%, 10 \%, 20 \%, 30 \%$ respectively, with blue lines denoting PIP2 and red lines denoting PS. The scale on the vertical axis is amplified 100-fold.

tangent component $\nabla_{s} \phi$ of the electrostatic field. Our simulations also show that other species of mobile ions such as $\mathrm{Ca}^{2+}, \mathrm{Mg}^{2+}$ will have similar screening effects. Note that the $\mathrm{Ca} / \mathrm{CaM}$ can also reverse the binding of MARCKS peptide to the membrane, so the current model needs to be integrated with models for $\mathrm{Ca} / \mathrm{CaM}$ association and $\mathrm{Ca} / \mathrm{CaM} / \mathrm{MARCKS}$ binding [53] to give a full description of the effects of $\mathrm{Ca}^{2+}$ on $\mathrm{PIP}_{2}$ sequestration.

\section{SUMMARY}

Lipid headgroups and membrane proteins diffuse laterally on the membrane surfaces. Local aggregation of lipids and proteins is usually a precursor of membrane fusion, fission, and budding and therefore is a critical step in many signaling pathways that are regulated by the membrane curvature. Lateral diffusion of charged headgroups are mediated by the nonspecific electrostatic interactions and hence can not be model by the conventional diffusion equation. Here we establish a surface electrodiffusion model with finite-size correction to describe the self-consistent lateral aggregation of charged lipid headgroups on curved membrane surfaces due to membrane/protein electrostatic interactions, without requiring the knowledge of locations of individual aggregated lipids. Our results agree very well

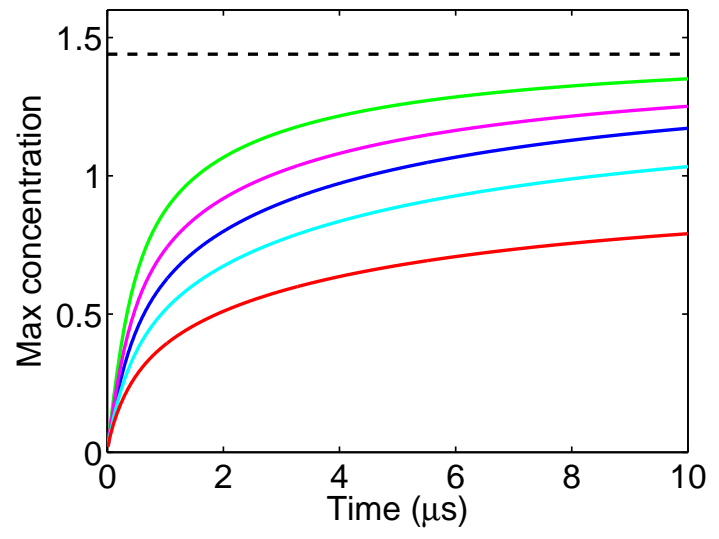

FIG. 5. Salt-dependent lipid sequestration. Maximum concentration of $\mathrm{PIP}_{2}$ decreases as salt concentration increases from $50 \mathrm{mM}$ (green), 100mM (magenta), 140mM (blue), $200 \mathrm{mM}$ (cyan) to $300 \mathrm{mM}$ (red). The number of sequestered $\mathrm{PIP}_{2}$ lipids are $4.02,3.35,2.89,2.16$ and 1.07 , respectively.

with the experimental observations and atomistic modeling of lipid sequestration. The ability to efficiently and accurately identify the local concentrations of specified proteins or lipid headgroups will enable us to integrate the selfconsistent lateral translocation of lipids and proteins to the analysis of membrane trafficking and curvature, and related signal pathways and diseases.

*yzhou@math.colostate.edu

[1] S. McLaughlin, J. Wang, A. Gambhir, and D. Murray, Annu. Rev. Biophys. Biomol. Struct., 31, 151 (2002).

[2] B.-C. Suh and B. Hille, Curr. Opin. Neurobiol., 15, 370 (2005).

[3] S. Höning, D. Ricotta, M. Krauss, K. Spte, B. Spolaore, A. Motley, M. Robinson, C. Robinson, V. Haucke, and D. J. Owen, Mol. Cell, 18, 519 (2005).

[4] P. A. Janmey and U. Lindberg, Nat. Rev. Mol. Cell Biol., 5, 658 (2004).

[5] P. D. Blood and G. A. Voth, Proc. Natl. Acad. Sci. U.S.A., 103, 15068 (2006).

[6] H. T. McMahon and J. L. Gallop, Nature, 438, 590 (2005).

[7] A. Hinderliter, P. F. F. Almeida, C. E. Creutz, and R. L. Biltonen, Biochemistry, 40, 4181 (2001), pMID: 11300799.

[8] S. McLaughlin and D. Murray, Nature, 438, 605 (2005).

[9] A. G. Petrov, Curr. Top. Membr, 58, 121 (2006).

[10] A. Filippov, G. Ordd, and G. Lindblom, Chemistry and Physics of Lipids, 159, 81 (2009).

[11] P. F. Fahey, D. E. Koppel, L. S. Barak, D. E. Wolf, E. L. Elson, and W. W. Webb, Science, 195, 305 (1977).

[12] K. Tanaka, P. A. Manning, V. K. Lau, and H. Yu, Langmuir, 15, 600 (1999).

[13] G. S. Ayton and G. A. Voth, Biophys. J., 87, 3299 (2004).

[14] A.-L. Kuo and C. G. Wade, Biochemistry, 18, 2300 (1979).

[15] J. F. Ellena, L. S. Lepore, and D. S. Cafiso, J. Phys. Chem., 97, 2952 (1993). 
[16] P. Cicuta, S. L. Keller, and S. L. Veatch, J. Phys. Chem. B, 111, 3328 (2007).

[17] Y. Chen, B. C. Lagerholm, B. Yang, and K. Jacobson, Methods, 39, 147 (2006), ISSN 1046-2023, analytical methods in the science of lipidomics, membrane organization and Protein-Lipid interactions.

[18] J. F. Tocanne, L. Dupou-Czanne, A. Lopez, and J. F. Tournier, FEBS Lett., 257, 10 (1989).

[19] W. L. C. Vaz and P. F. F. Almeida, Biophys. J., 60, 1553 (1991).

[20] I. Vattulainen and O. G. Mouritsen, in Diffusion in Condensed Matter: Methods, Materials, Models (SpringerVerlag, 2005) pp. 471-509.

[21] M. Cohen and D. Turnbull, J. Chem. Phys., 31, 1164 (1959).

[22] D. Turnbull and M. Cohen, J. Chem. Phys., 34, 120 (1961).

[23] J. E. MacCarthy and J. J. Kozak, J. Chem. Phys., 77, 2214 (1982).

[24] M. J. Saxton, Biophys. J., 39, 165 (1982).

[25] J. L. Lebowitz, E. Helfand, and E. Praestgaard, J. Chem. Phys., 43, 774 (1965).

[26] T. J. O'leary, Proc. Natl. Acad. Sci. U.S.A., 84, 429 (1987).

[27] E. Falck, T. Rg, M. Karttunen, and I. Vattulainen, J. Am. Chem. Soc., 130, 44 (2008).

[28] C. Amatore, A. I. Oleinick, O. V. Klymenko, and I. Svir, Chemphyschem., 10, 1586 (2009).

[29] C. Amatore, O. V. Klymenko, A. I. Oleinick, and I. Svir, Chemphyschem., 10, 1593 (2009).

[30] T. Yoshigaki, Phys. Rev. E, 75, 041901 (2007).

[31] S. M. Leitenberger, E. Reister-Gottfried, and U. Seifert, Langmuir, 24, 1254 (2008).

[32] W. T. Góźdź, Langmuir, 24, 12458 (2008),

[33] C. Chevalier and F. Debbasch, Europhys. Lett., 77, 20005 (2007).

[34] M. S. Kilic, M. Z. Bazant, and A. Ajdari, Phys. Rev. E, 75, 021503 (2007).

[35] B. Lu and Y. C. Zhou, Biophys. J., 100, 2475 (2011).
[36] V. Y. Kiselev, M. Leda, A. I. Lobanov, D. Marenduzzo, and A. B. Goryachev, J. Chem. Phys., 135, 155103 (2011),

[37] V. Y. Kiselev, D. Marenduzzo, and A. B. Goryachev, Biophys. J., 100, 1261 (2011).

[38] G. Khelashvill, H. Weinstein, and D. Harries, Biophys. J., 94, 2580 (2008).

[39] I. Borukhov, D. Andelman, and H. Orland, Phys. Rev. Lett., 79, 435 (1997).

[40] Y. Burak and D. Andelman, J. Chem. Phys., 114, 3271 (2001).

[41] J.-P. Hansen and I. R. McDonald, Theory of simple liquids (Academic Press, 2006).

[42] H. A. Stone, Phys. Fluids A, 2, 111 (1990).

[43] G. Dziuk, in Partial differential equations and calculus of variations (Springer, Berlin, 1988).

[44] A. Arbuzova, A. A. P. Schmitz, and G. Verères, Biochem. J., 15, 1 (2002).

[45] T. V. Ratto and M. L. Longo, Biophys. J., 83, 3380 (2002).

[46] P. Shorten and J. Sneyd, Biophys. J., 96, 4764 (2009).

[47] E. Flenner, J. Das, M. C. Rheinstädter, and I. Kosztin, Phys. Rev. E, 79, 011907 (2009).

[48] Y. C. Zhou, B. Lu, and A. A. Gorfe, Phys. Rev. E, 82, 041923 (2010).

[49] J. Wang, A. Gambhir, G. H.-M. lyne, D. Murray, U. Golebiewska, and S. McLaughlin, J. Biol. Chem., 277, 34401 (2002).

[50] J. Wang, A. Gambhir, S. McLaughlin, and D. Murray, Biophys. J., 86, 1969 (2004).

[51] U. Golebiewska, A. Gambhir, G. Hangys-Mihlyn, I. Zaitseva, J. Rdler, and S. McLaughlin, Biophys. J., 91, 588 (2006), ISSN 0006-3495.

[52] A. Arbuzova, D. Murray, and S. McLaughlin, Biochimica et Biophysica Acta (BBA) - Reviews on Biomen branes, 1376, 369 (1998).

[53] J. Kim, T. Shishido, X. Jiang, A. Aderem, and S. McLaughlin, J. Biol. Chem., 269, 28214 (1994). 